\title{
Between Strassen and Chung normalizations for Lévy's area process*
}

\author{
MODESTE N'ZI ${ }^{1}$, BRUNO RÉMILLARD ${ }^{2}$ and RADU THEODORESCU ${ }^{*}$ \\ ${ }^{1}$ Département de Mathématiques, Université d'Abidjan, 22 BP, 582 Abidjan 22, Côte d'Ivoire, \\ ${ }^{2}$ Département de Mathématiques et d'Informatique, Université du Québec à Trois-Rivières, CP \\ 500, Trois-Rivières, Québec, Canada G9A $5 H 7$ \\ ${ }^{3}$ Département de Mathématiques et de Statistique, Université Laval, Sainte-Foy, Québec, Canada \\ G1K $7 P 4$
}

Let $\{L(t): t \geqslant 0\}$ be Lévy's area process, let $\gamma: \mathbf{R}_{+} \mapsto \mathbf{R}$, and let $\left\{Z_{t}: t \geqslant 3\right\}$ be the stochastic process defined by $Z_{t}(s)=L(t s) /(2 t \log \log t), 0 \leqslant s \leqslant 1$. Conditions on $\gamma$ are given such that the set of all limit points of $\left\{\gamma(t) Z_{t}: t \geqslant 3\right\}$ as $t \rightarrow \infty$ is a.s. equal to the set of all continuous functions defined on $[0,1]$ which vanish at 0 .

Keywords: Brownian motion; law of the iterated logarithm; Lévy's area process

1350-7265 (C) 1998 Chapman \& Hall

\section{Introduction}

Let $B=\{B(t): t \geqslant 0\}$ be an $m$-dimensional Brownian motion, let $\gamma: \mathbf{R}_{+} \mapsto \mathbf{R}$, let $\mathscr{C}_{0}^{m}$ denote the set of all $\mathbf{R}^{m}$-valued continuous functions defined on $[0,1]$ which vanish at 0 , endowed with the uniform topology, and let

$$
\phi(t)= \begin{cases}1, & \text { for } 0<t<3, \\ \log \log t, & \text { for } t \geqslant 3\end{cases}
$$

Let us consider the following conditions.

(A) $\gamma(t) \rightarrow \infty$ as $t \rightarrow \infty$.

$\left(\mathrm{B} \delta_{0}\right)$ There exist $M>0$ and $0<\delta<\delta_{0} \leqslant 2$ such that $\gamma(t) \leqslant M \phi^{\delta}(t)$ for $t$ sufficiently large.

Baldi and Roynette (1992a) proved the following result:

Theorem 1.1. Assume (A) and (B1), and set

$$
X_{t}(s)=\frac{B(t s)}{\{2 t \phi(t)\}^{1 / 2}}, \quad 0 \leqslant s \leqslant 1, \quad t>0 .
$$

Then the set of all limit points of $\left\{\gamma(t) X_{t}: t>0\right\}$ is a.s. equal to $\mathscr{C}_{0}^{m}$.

*To whom correspondence should be addressed. e-mail: RADUTHEO@MAT.ULAVAL.CA. 
Consider now Lévy's (1949) area process $L=\{L(t): t \geqslant 0\}$ associated with a twodimensional Brownian motion $B=\left(B_{1}, B_{2}\right)$ :

$$
L(t)=\frac{1}{2}\left(\int_{0}^{t} B_{1}(u) B_{2}(\mathrm{~d} u)-\int_{0}^{t} B_{2}(u) B_{1}(\mathrm{~d} u)\right), \quad t \geqslant 0,
$$

as the stochastic analogue of the area contained in a lens-shaped domain. Some applications of this process in analysis and in statistical inference can be found in the work of Gaveau (1977) and Liptser and Shiryaev (1977, p. 212), respectively. For $L$ the classical law of the iterated logarithm (see, for example, Berthuet 1979) as well as its functional form (see, for example, Baldi 1986 and Helmes et al. 1986) have been proved. Chung's version of the law of the iterated logarithm for the area process and related processes was recently established by Rémillard (1994). In view of these results and since the area process shares many properties with the linear Brownian motion (see, for example, Helmes 1986), one would expect that an analogue of Theorem 1.1 holds for $L$. The aim of this article is to confirm this assertion. More precisely, we prove the following main result.

Theorem 1.2. Assume (A) and (B2), and set

$$
Z_{t}(s)=\frac{L(t s)}{2 t \phi(t)}, \quad 0 \leqslant s \leqslant 1, \quad t>0 .
$$

Then the set of all limit points of $\left\{\gamma(t) Z_{t}: t>0\right\}$ is a.s. equal to $\mathscr{C}_{0}^{1}$.

Remark 1.3. (a) The classical law of the iterated logarithm states that $[-1 / \pi, 1 / \pi]$ is a.s. the set of all limit points of $\left\{Z_{t}(1): t>0\right\}$ as $t \rightarrow \infty$. Therefore, for every function $\gamma$ such that $\gamma(t) \rightarrow \infty$ as $t \rightarrow \infty$, the set of all limit points of $\left\{\gamma(t) Z_{t}(1): t>0\right\}$ is a.s. equal to $\mathbf{R}$. Thus Theorem 1.2 is the analogue of this result when the classical law is replaced by that of Strassen.

(b) Theorem 1.2 is optimal since Chung's form of the law of the iterated logarithm in Rémillard (1994) says that a.s.

$$
\liminf _{t \rightarrow \infty} \frac{\phi(t)}{2 t} \sup _{0 \leqslant s \leqslant 1}|L(t s)|=\frac{\pi}{8}
$$

It follows from (2) that, if $\gamma(t) \sim \phi^{2}(t)$ as $t \rightarrow \infty$, then every point within the ball in $\mathscr{C}_{0}^{1}$ of radius $\pi / 8$ and centre 0 is not a limit point of $\left\{\gamma(t) Z_{t}: t>0\right\}$.

In Section 2 we give three auxiliary lemmas which we need for the proof of our main result in Section 3.

In what follows we shall use the following notation: $|\cdot|$, Euclidean norm in $\mathbf{R}^{m}$; $\|\cdot\|$, the uniform norm in $\mathscr{C}_{0}^{m} ;\langle\cdot, \cdot\rangle$, the scalar product;

$$
H^{m}=\left\{f \in \mathscr{C}_{0}^{m}: f(s)=\int_{0}^{s} \dot{f}(u) \mathrm{d} u \text { with } \int_{0}^{1}|\dot{f}(u)|^{2} \mathrm{~d} u<\infty\right\} .
$$


Further let $\mu: \mathscr{C}_{0}^{m} \mapsto \overline{\mathbf{R}}_{+}$be the application defined by

$$
\mu(f)= \begin{cases}\frac{1}{2} \int_{0}^{1}|\dot{f}(u)|^{2} \mathrm{~d} u, & \text { for } f \in H^{m} \\ \infty, & \text { otherwise }\end{cases}
$$

and let $F: H^{2} \mapsto \mathscr{C}_{0}^{1}$ be the functional associated with the area process: for every $f=\left(f_{1}, f_{2}\right) \in H^{2}$,

$$
F(f)(t)=\frac{1}{2}\left(\int_{0}^{t} f_{1}(u) \dot{f}_{2}(u) \mathrm{d} u-\int_{0}^{t} f_{2}(u) \dot{f}_{1}(u) \mathrm{d} u\right), \quad t \geqslant 0 .
$$

Let us also consider the application $\lambda: \mathscr{C}_{0}^{1} \mapsto \overline{\mathbf{R}}_{+}$such that

$$
\lambda(g)= \begin{cases}\inf \{\mu(f): F(f)=g\}, & \text { for } F^{-1}(\{g\}) \neq \varnothing, \\ \infty, & \text { otherwise. }\end{cases}
$$

It is known that, if $\lambda(g)<\infty$, then there exists $f \in H^{2}$ such that $F(f)=g$ and $\mu(f)=\lambda(g)$ (see, for example, Azencott 1980, p. 69).

Finally, observe that, if we consider the skew-symmetric matrix

$$
J=\left(\begin{array}{cc}
0 & -0.5 \\
0.5 & 0
\end{array}\right)
$$

then (1) becomes

$$
L(t)=\int_{0}^{t}\langle J B(u), B(\mathrm{~d} u)\rangle, \quad t \geqslant 0 .
$$

\section{Auxiliary results}

We begin with the following lemma.

Lemma 2.1. Let $g \in \mathscr{C}_{0}^{1}$ such that $\lambda(g)=\mu(f)<\infty$ for some $f$ in $H^{2}$. Then, for any $r>0$, we have

$$
P(\|L-g\| \leqslant r) \geqslant \mathrm{e}^{-\lambda(g)} P\left(\|L\| \leqslant \frac{r}{2},\|B\| \leqslant \frac{r}{2 c(f)}\right),
$$

where $c(f)=3\{2 \mu(f)\}^{1 / 2}$.

Proof. Let $f \in H^{2}$ be such that $F(f)=g$ and $\mu(f)=\lambda(g)$. Set $\tilde{B}=B-f$. By virtue of Girsanov's theorem, $\tilde{B}$ is a two-dimensional Brownian motion under the probability measure $\tilde{Q}$ defined by the Radon-Nikodym derivative

$$
\frac{\mathrm{d} P}{\mathrm{~d} \tilde{Q}}=\exp \left(-\int_{0}^{1}\langle\dot{f}(s), \tilde{B}(\mathrm{~d} s)\rangle-\mu(f)\right) .
$$


Set

$$
\tilde{X}(t)=2 \int_{0}^{t}\langle J \tilde{B}(s), \dot{f}(s)\rangle \mathrm{d} s-\langle J \tilde{B}(t), f(t)\rangle .
$$

Then $\|\tilde{X}\| \leqslant c(f)\|\tilde{B}\|$. Moreover,

$$
\tilde{X}(t)=\int_{0}^{t}\langle J \tilde{B}(s), \dot{f}(s)\rangle \mathrm{d} s+\int_{0}^{t}\langle J f(s), \tilde{B}(\mathrm{~d} s)\rangle, \quad \text { for all } t \geqslant 0, \quad \tilde{Q} \text { a.s., }
$$

and

$$
L(t)-g(t)=\tilde{L}(t)+\tilde{X}(t), \quad \text { for all } t \geqslant 0, \quad \tilde{Q} \text { a.s. }
$$

where

$$
\tilde{L}(t)=\int_{0}^{t}\langle J \tilde{B}(s), \tilde{B}(\mathrm{~d} s)\rangle
$$

Hence

$$
\|L-g\|=\|\tilde{L}+\tilde{X}\| \leqslant\|\tilde{L}\|+c(f)\|\tilde{B}\|
$$

Consequently

$$
\begin{aligned}
& P(\|L-g\| \leqslant r) \geqslant P\left(\|\tilde{L}\| \leqslant \frac{r}{2},\|\tilde{B}\| \leqslant \frac{r}{2 c(f)}\right) \\
& =\mathrm{e}^{-\lambda(g)} \int 1_{\{\|\tilde{L}\| \leqslant r / 2,\|\tilde{B}\| \leqslant r / 2 c(f)\}} \mathrm{e}^{-\int_{0}^{1}\langle\dot{f}(s), \tilde{B}(\mathrm{~d} s)\rangle} \mathrm{d} \tilde{Q} \\
& =\mathrm{e}^{-\lambda(g)} \int 1_{\{\|L\| \leqslant r / 2,\|B\| \leqslant r / 2 c(f)\}} \mathrm{e}^{-\int_{0}^{1}\langle\dot{f}(s), B(\mathrm{~d} s)\rangle} \mathrm{d} P,
\end{aligned}
$$

since $\tilde{B}$ is a two-dimensional Brownian motion with respect to $\tilde{Q}$.

The last chain of inequalities also holds for $-f$. Since $c(-f)=c(f)$ and $\cosh x \geqslant 1$ for every real $x$, it follows that

$$
\begin{aligned}
P(\|L-g\| \leqslant r) \geqslant & \frac{1}{2} \mathrm{e}^{-\lambda(g)} \int 1_{\{\|L\| \leqslant r / 2,\|B\| \leqslant r / 2 c(f)\}} \mathrm{e}^{-\int_{0}^{1}\langle\dot{f}(s), B(\mathrm{~d} s)\rangle} \mathrm{d} P \\
& +\frac{1}{2} \mathrm{e}^{-\lambda(g)} \int 1_{\{\|L\| \leqslant r / 2,\|B\| \leqslant r / 2 c(-f)\}} \mathrm{e}^{-\int_{0}^{1}\langle-\dot{f}(s), B(\mathrm{~d} s)\rangle} \mathrm{d} P \\
= & \mathrm{e}^{-\lambda(g)} \int 1_{\{\|L\| \leqslant r / 2,\|B\| \leqslant r / 2 c(f)\}} \cosh \left(\int_{0}^{1}\langle\dot{f}(s), B(\mathrm{~d} s)\rangle\right) \mathrm{d} P \\
\geqslant & \mathrm{e}^{-\lambda(g)} P\left(\|L\| \leqslant \frac{r}{2},\|B\| \leqslant \frac{r}{2 c(f)}\right) .
\end{aligned}
$$

The next result is inspired by Lemma 2.3 of Rémillard (1994). 
Lemma 2.2. Suppose that $0<l<\infty$ and $0<h \leqslant \infty$. Then there exist two finite positive constants $\delta_{1}$ and $\delta_{2}$ such that

$$
P\left\{\sup _{0 \leqslant s \leqslant t}|L(s)|<l, \sup _{0 \leqslant s \leqslant t}|B(s)|<h\right\} \geqslant \delta_{1} \mathrm{e}^{-\delta_{2} t} .
$$

Proof. For any infinitely differentiable function $u$ on $\mathbf{R}^{2+1}$, let

$$
\mathscr{B} u(x, y)=\frac{1}{2} \Delta_{x} u(x, y)+\frac{1}{8}|x|^{2} \partial_{y}^{2} u(x, y)+\left\langle J x, \nabla_{x} \partial_{y} u(x, y)\right\rangle .
$$

$\mathscr{B}$ is the generator of the diffusion process $\{B(t), L(t): t \geqslant 0\}$ (see, for example, Rémillard 1994); it is known as the Kohn-Laplace differential operator (see, for example, Gaveau 1977, p. 98).

Now, for any $n \geqslant 0$, let $R_{n}$ be the simple Laguerre polynomial, i.e.,

$$
R_{n}(t)=\sum_{j=0}^{n}\left(\begin{array}{l}
n \\
j
\end{array}\right) \frac{(-t)^{j}}{j !}
$$

Then $R_{n}$ satisfies the differential equation

$$
t R_{n}^{\prime \prime}(t)+(1-t) R_{n}^{\prime}(t)+n R_{n}(t)=0, \quad \text { for all } t \in \mathbf{R},
$$

and let $z_{n}$ be the smallest positive zero of $R_{n}$. Then $z_{n} \rightarrow 0$ as $n \rightarrow \infty$ because

$$
\lim _{n \rightarrow \infty} R_{n}\left(\frac{t}{n}\right)=\sum_{j=0}^{\infty} \frac{(-t)^{j}}{(j !)^{2}}=J_{0}\left(2 t^{1 / 2}\right),
$$

where $J_{0}$ is the Bessel function of the first kind of order 0 . In fact, if $a$ is the smallest positive zero of $J_{0}$ and if $0<a_{1}<a<a_{2}$ are chosen such that $J_{0}\left(a_{1}\right)>0$ and $J_{0}\left(a_{2}\right)<0$, it follows that $R_{n}\left(a_{1}^{2} / 4 n\right)>0$ and $R_{n}\left(a_{2}^{2} / 4 n\right)<0$ for $n$ large enough. Hence there is at least one zero in the interval $\left(a_{1}^{2} / 4 n, a_{2}^{2} / 4 n\right)$ proving that $z_{n}<a_{2}^{2} / 4 n$. Moreover, $R_{n}(t)>0$ for all $t \in\left[0, z_{n}\right)$. Set $a_{n}=\sup _{0 \leqslant t \leqslant z_{n}} R_{n}(t)$.

Further, if $\theta=\pi / 4 l$, the function

$$
u_{n}(x, y)=\mathrm{e}^{-\theta|x|^{2} / 2} R_{n}\left(\theta|x|^{2}\right) \cos (\theta y)
$$

satisfies the equation

$$
\mathscr{B} u_{n}=-\theta(2 n+1) u_{n}
$$

for any $(x, y) \in \mathbf{R}^{2+1}$. Since $R_{n}$ is a polynomial of degree $n$, there exists a constant $D_{n}$ such that $\left|R_{n}(t)\right| \leqslant D_{n}|t|^{n}$, such that $\left|u_{n}(x, y)\right| \leqslant D_{n} \theta^{n}|x|^{2 n} \mathrm{e}^{-\theta|x|^{2} / 2}$. Therefore $M_{n}(t)=$ $\mathrm{e}^{t \theta(2 n+1)} u_{n}\left(B_{t}, L_{t}\right)$ is integrable and is a martingale. Moreover, $u_{n}(x, y)=0$ if $|y|=l$ or if $|x|=h_{n}=\left(z_{n} / \theta\right)^{1 / 2}$.

Let now $0<h \leqslant \infty$ and $l>0$ be given and let $C=C(l, h)$ be the cylinder:

$$
C(l, h)=\left\{(x, y) \in \mathbf{R}^{2+1}:|x| \leqslant h,|y| \leqslant l\right\} .
$$

Next, let $T_{l, h}=\inf \left\{t>0:\left(B_{t}, L_{t}\right) \notin C(l, h)\right\}$. $T_{l, h}$ is a stopping time. Our aim is to show that there exist two finite positive constants $\delta_{1}$ and $\delta_{2}$ such that

$$
P\left(T_{l, h}>t\right) \geqslant \delta_{1} \mathrm{e}^{-\delta_{2} t} .
$$


Indeed, choose $n$ sufficiently large that $h_{n}=\left(z_{n} / \theta\right)^{1 / 2} \leqslant h$. If $h=\infty$, take $n=0$ and in this case $z_{0}=+\infty$. For simplicity, set $T_{n}=T_{l, h_{n}}$. Then $M_{n}\left(\min \left\{t, T_{n}\right\}\right)$ is a martingale and

$$
1=M_{n}(0)=E\left(M_{n}\left(\min \left\{t, T_{n}\right\}\right)\right)=E\left(\mathrm{e}^{t \theta(2 n+1)} u_{n}\left(B_{t}, L_{t}\right) 1_{\left\{T_{n}>t\right\}}\right),
$$

since $M_{n}\left(T_{n}\right)=0$ if $T_{n} \leqslant t$. Therefore

$$
1 \leqslant \mathrm{e}^{t \theta(2 n+1)} \sup _{(x, y) \in C\left(l, h_{n}\right)} u_{n}(x, y) P\left(T_{n}>t\right) \leqslant a_{n} \mathrm{e}^{t \theta(2 n+1)} P\left(T_{n}>t\right),
$$

yielding

$$
P\left(T_{n}>t\right) \geqslant a_{n}^{-1} \mathrm{e}^{-t \theta(2 n+1)}
$$

and the proof is completed.

Next, for every integer $k \geqslant 2$, define $t_{k}=k^{k}$ and let $L^{k}$ be the area process associated with the two-dimensional Brownian motion $B^{k}=\left\{B^{k}(s)=B\left(s+t_{k-1}\right)-B\left(t_{k-1}\right): s \geqslant 0\right\}$. Lemmas 2.1 and 2.2 are used to prove the following.

Lemma 2.3. For every $r>0$, every integer $k \geqslant 2$, and every $g \in \mathscr{C}_{0}^{1}$ such that $\lambda(g)<\infty$, let $C_{k}^{r}$ denote the event

$$
\left\{\sup _{0 \leqslant s \leqslant\left(t_{k}-t_{k-1}\right) / t_{k}}\left|\gamma\left(t_{k}\right) \frac{L^{k}\left(t_{k} s\right)}{2 t_{k} \phi\left(t_{k}\right)}-g(s)\right| \leqslant r\right\}
$$

Then

$$
\liminf _{k \rightarrow \infty} \frac{1}{\phi\left(t_{k}\right)} \log P\left(C_{k}^{r}\right)=0
$$

In particular $\sum_{k} P\left(C_{k}^{r}\right)=\infty$.

Proof. We have

$$
P\left(C_{k}^{r}\right) \geqslant P\left(\left\|\gamma\left(t_{k}\right) \frac{L^{k}\left(t_{k} \cdot\right)}{2 t_{k} \phi\left(t_{k}\right)}-g\right\| \leqslant r\right) .
$$

Since $L^{k}\left(t_{k} \cdot\right) / t_{k}$ has the same law as $L$ (see, for example, Proposition 2.1 of Helmes 1986), we deduce that

$$
P\left(C_{k}^{r}\right) \geqslant P\left(\left\|\gamma\left(t_{k}\right) \frac{L}{2 \phi\left(t_{k}\right)}-g\right\| \leqslant r\right)=P\left(\left\|L-\frac{2 \phi\left(t_{k}\right)}{\gamma\left(t_{k}\right)} g\right\| \leqslant \frac{2 \phi\left(t_{k}\right)}{\gamma\left(t_{k}\right)} r\right) .
$$

For simplicity, set $\theta_{k}=2 \phi\left(t_{k}\right) / \gamma\left(t_{k}\right)$. Let $f \in H^{2}$ be such that $F(f)=g$ and $\lambda(g)=$ $\mu(f)$. Then $F\left(\theta_{k}^{1 / 2} f\right)=\theta_{k} g, \lambda\left(\theta_{k} g\right)=\theta_{k} \lambda(g)$ and $c\left(\theta_{k}^{1 / 2} f\right)=\theta_{k}^{1 / 2} c(f)$. Since the law of 
$\{(B(s t), L(s t)): s \geqslant 0\}$ is the same as that of $\left\{\left(t^{1 / 2} B(s), t L(t)\right): s \geqslant 0\right\}$ for any $t>0$ and using also Lemma 2.1, and Lemma 2.2 with $t=1 / \theta_{k}, l=r / 2$ and $h=r / 2 c(f)$, we obtain

$$
\begin{aligned}
P\left(C_{k}^{r}\right) & \geqslant \mathrm{e}^{-\theta_{k} \lambda(g)} P\left(\|L\| \leqslant \frac{\theta_{k} r}{2},\|B\| \leqslant \frac{\theta_{k}^{1 / 2} r}{2 c(f)}\right) \\
& =\mathrm{e}^{-\theta_{k} \lambda(g)} P\left\{\sup _{0 \leqslant s \leqslant 1 / \theta_{k}}|L(s)| \leqslant \frac{r}{2}, \sup _{0 \leqslant s \leqslant 1 / \theta_{k}}|B(s)| \leqslant \frac{r}{2 c(f)}\right\} \\
& \geqslant \mathrm{e}^{-\theta_{k} \lambda(g)} \delta_{1} \mathrm{e}^{-\delta_{2} / \theta_{k}} .
\end{aligned}
$$

Therefore, in view of Conditions (A) and (B2), we obtain

$$
\liminf _{k \rightarrow \infty} \frac{1}{\phi\left(t_{k}\right)} \log P\left(C_{k}^{r}\right) \geqslant \liminf _{k \rightarrow \infty}\left(\frac{-2 \lambda(g)}{\gamma\left(t_{k}\right)}+\frac{\log \delta_{1}}{\phi\left(t_{k}\right)}-\frac{\delta_{2} \gamma\left(t_{k}\right)}{2 \phi\left(t_{k}\right)^{2}}\right)=0 .
$$

Thus

$$
\liminf _{k \rightarrow \infty} \frac{1}{\phi\left(t_{k}\right)} \log P\left(C_{k}^{r}\right)=0 .
$$

Hence, if $k$ is large enough,

$$
P\left(C_{k}^{r}\right) \geqslant \mathrm{e}^{-0.5 \phi\left(t_{k}\right)}=\frac{1}{(k \log k)^{1 / 2}},
$$

proving that $\sum_{k} P\left(C_{k}^{r}\right)=\infty$.

\section{Proof of the main result}

We now prove Theorem 1.2. At a certain moment we shall use Lemma 2.3.

Since $H^{1}$ is dense in $\mathscr{C}_{0}^{1}$, it suffices to prove that the set of all limit points of $\left\{\gamma(t) Z_{t}: t>0\right\}$ contains a.s. $H^{1}$. To this purpose we only have to show that, for every $r>0$ and every $g \in H^{1}$,

$$
P\left(\liminf _{t \rightarrow \infty}\left\|\gamma(t) Z_{t}-g\right\| \leqslant r\right)=1
$$

First we prove that

$$
P\left(\liminf _{k \rightarrow \infty}\left\|\gamma\left(t_{k}\right) Z_{t_{k}}-g\right\| \leqslant r\right)=1 .
$$

Let us note that

$$
\begin{aligned}
\left\|\gamma\left(t_{k}\right) Z_{t_{k}}-g\right\| & \leqslant \sup _{0 \leqslant s \leqslant t_{k-1} / t_{k}}\left|\gamma\left(t_{k}\right) Z_{t_{k}}(s)-g(s)\right|+\sup _{t_{k-1} / t_{k} \leqslant s \leqslant 1}\left|\gamma\left(t_{k}\right) Z_{t_{k}}(s)-g(s)\right| \\
& =I_{1}^{k}+I_{2}^{k} .
\end{aligned}
$$


We have

$$
I_{1}^{k}=\sup _{0 \leqslant s \leqslant 1}\left|\gamma\left(t_{k}\right) \frac{L\left(t_{k-1} s\right)}{2 t_{k} \phi\left(t_{k}\right)}-g\left(\frac{t_{k}}{t_{k-1}} s\right)\right| \leqslant \gamma\left(t_{k}\right)\left\|\frac{L\left(t_{k-1} \cdot\right)}{2 t_{k} \phi\left(t_{k}\right)}\right\|+\left\|g\left(\frac{t_{k}}{t_{k-1}} \cdot\right)\right\| .
$$

Therefore

$$
I_{1}^{k} \leqslant \gamma\left(t_{k}\right) \frac{t_{k-1}}{t_{k}} \frac{\phi\left(t_{k-1}\right)}{\phi\left(t_{k}\right)}\left\|\frac{L\left(t_{k-1} \cdot\right)}{2 t_{k-1} \phi\left(t_{k-1}\right)}\right\|+\left(\frac{t_{k-1}}{t_{k}}\right)^{1 / 2}\left(\int_{0}^{1}|\dot{g}(u)|^{2} \mathrm{~d} u\right)^{1 / 2} .
$$

In view of Condition (A) and since $t_{k-1} / t_{k} \leqslant 1 / k$, there exists $0<C_{1}<\infty$ such that, for $k$ large enough,

$$
I_{1}^{k} \leqslant \frac{C_{1}(\log k)^{\delta}}{k}\left\|\frac{L\left(t_{k-1} \cdot\right)}{2 t_{k-1} \phi\left(t_{k-1}\right)}\right\|+\frac{1}{k^{1 / 2}}\left(\int_{0}^{1}|\dot{g}(u)| \mathrm{d} u\right)^{1 / 2}
$$

By virtue of Strassen's law of the iterated logarithm for the area process, the sequence

$$
\left\{\left\|\frac{L\left(t_{k-1} \cdot\right)}{2 t_{k-1} \phi\left(t_{k-1}\right)}\right\|: k \geqslant 2\right\}
$$

is bounded. Combining this fact with (6), we obtain that

$$
\lim _{k \rightarrow \infty} I_{1}^{k}=0
$$

By virtue of (7), the proof of (5) reduces to that of $P\left(\left(\liminf \operatorname{in}_{k \rightarrow \infty} I_{2}^{k} \leqslant r\right)=1\right.$.

We have

$$
\begin{aligned}
I_{2}^{k} & =\sup _{t_{k-1} / t_{k} \leqslant s \leqslant 1}\left|\gamma\left(t_{k}\right) \frac{L\left(t_{k} s\right)}{2 t_{k} \phi\left(t_{k}\right)}-g(s)\right|=\sup _{0 \leqslant s \leqslant t_{k}-t_{k-1}}\left|\gamma\left(t_{k}\right) \frac{L\left(s+t_{k-1}\right)}{2 t_{k} \phi\left(t_{k}\right)}-g\left(\frac{s+t_{k-1}}{t_{k}}\right)\right| \\
& \leqslant \sup _{0 \leqslant s \leqslant t_{k}-t_{k-1}}\left|\gamma\left(t_{k}\right) \frac{L\left(s+t_{k-1}\right)-L\left(t_{k-1}\right)}{2 t_{k} \phi\left(t_{k}\right)}-g\left(\frac{s+t_{k-1}}{t_{k}}\right)\right|+\gamma\left(t_{k}\right)\left|\frac{L\left(t_{k-1}\right)}{2 t_{k} \phi\left(t_{k}\right)}\right| \\
& =J_{1}^{k}+J_{2}^{k} .
\end{aligned}
$$

By similar arguments as the proof of (7), one may prove that $\lim _{k \rightarrow \infty} J_{2}^{k}=0$. Therefore, it remains to prove that $P\left(\liminf _{k \rightarrow \infty} J_{1}^{k} \leqslant r\right)=1$.

Now, by virtue of Proposition 2.1 of Helmes (1986), we have

$$
L\left(s+t_{k-1}\right)-L\left(t_{k-1}\right)=L^{k}(s)+\left\langle J B\left(t_{k-1}\right), B^{k}(s)\right\rangle, \quad s \geqslant 0 .
$$

It follows that 


$$
\begin{aligned}
J_{1}^{k} \leqslant & \sup _{0 \leqslant s \leqslant t_{k}-t_{k-1}}\left|\gamma\left(t_{k}\right) \frac{L^{k}(s)}{2 t_{k} \phi\left(t_{k}\right)}-g\left(\frac{s}{t_{k}}\right)\right|+\sup _{0 \leqslant s \leqslant t_{k}-t_{k-1}}\left|\gamma\left(t_{k}\right) \frac{\left\langle J B\left(t_{k-1}\right), B^{k}(s)\right\rangle}{2 t_{k} \phi\left(t_{k}\right)}\right| \\
& +\sup _{0 \leqslant s \leqslant t_{k}-t_{k-1}}\left|g\left(\frac{s}{t_{k}}\right)-g\left(\frac{s+t_{k-1}}{t_{k}}\right)\right| \\
= & K_{1}^{k}+K_{2}^{k}+K_{3}^{k} .
\end{aligned}
$$

By virtue of the Cauchy-Schwarz inequality, we have

$$
K_{3}^{k} \leqslant\left(\frac{t_{k-1}}{t_{k}}\right)^{1 / 2}\left(\int_{0}^{1}|\dot{g}(u)|^{2} \mathrm{~d} u\right)^{1 / 2} .
$$

So, $\lim _{k \rightarrow \infty} K_{3}^{k}=0$.

Now, let $0<r_{1}<r$ and consider the events

$$
\begin{aligned}
& A_{k}=\left\{\sup _{0 \leqslant s \leqslant t_{k}-t_{k-1}} \gamma\left(t_{k}\right)\left|\frac{\left\langle J B\left(t_{k-1}\right), B^{k}(s)\right\rangle}{2 t_{k} \phi\left(t_{k}\right)}\right| \leqslant r-r_{1}\right\}, \\
& B_{k}=\left\{\sup _{0 \leqslant s \leqslant t_{k}-t_{k-1}}\left\{\phi\left(t_{k}\right)\right\}^{2}\left|\frac{\left\langle J B\left(t_{k-1}\right), B^{k}(s)\right\rangle}{2 t_{k} \phi\left(t_{k}\right)}\right| \leqslant \frac{r-r_{1}}{M}\right\}, \\
& C_{k}=\left\{\sup _{0 \leqslant s \leqslant t_{k}-t_{k-1}}\left|\gamma\left(t_{k}\right) \frac{L^{k}(s)}{2 t_{k} \phi\left(t_{k}\right)}-g\left(\frac{s}{t_{k}}\right)\right| \leqslant r_{1}\right\}, \\
& D_{k}=\left\{K_{1}^{k}+K_{2}^{k} \leqslant r\right\} .
\end{aligned}
$$

In view of Condition (B2), for $k$ large enough, $B_{k} \subset A_{k}$. It follows that $P\left(A_{k}^{c}\right.$ i.o. $) \leqslant P\left(B_{k}^{c}\right.$ i.o.). By virtue of Lemma 3.2 of Rémillard (1994), we have $P\left(B_{k}^{c}\right.$ i.o. $)=0$. Therefore $P\left(A_{k}^{c}\right.$ i.o. $)=0$. Now, $A_{k} \cap C_{k}$ being a subset of $D_{k}$, we have $P\left(A_{k} \cap C_{k}\right.$ i.o. $) \leqslant P\left(D_{k}\right.$ i.o. $)$. Moreover $P\left(A_{k}^{c}\right.$ i.o. $)=0$. It follows that

$$
P\left(D_{k} \text { i.o. }\right) \geqslant P\left(C_{k} \text { i.o. }\right) \text {. }
$$

Next, let $\mathscr{F}_{t}=\sigma\left(B_{s}: 0 \leqslant s \leqslant t\right)$. It is easy to see that the event $C_{k}$ belongs to $\mathscr{F}_{t_{k}}$ and is independent of $\mathscr{F}_{t_{k-1}}$. By virtue of Lemma 2.3 and Lévy's extension of the Borel-Cantelli lemma (Lévy 1954, Corollary 68, p. 249), we deduce that $P\left(C_{k}\right.$ i.o. $)=1$. Therefore, by using $(8)$, we obtain $P\left(D_{k}\right.$ i.o. $)=1$ which implies that $P\left(\liminf \operatorname{int}_{k \rightarrow \infty} J_{1}^{k} \leqslant r\right)=1$. Thus we proved (5). Since

$$
\left.P\left(\liminf _{k \rightarrow \infty} \| \gamma\left(t_{k}\right) Z_{t_{k}}-g\right) \| \leqslant r\right) \leqslant P\left(\liminf _{t \rightarrow \infty}\left\|\gamma(t) Z_{t}-g\right\| \leqslant r\right)
$$

we are led to (4). By letting $r \rightarrow 0$ in (4), we obtain 


$$
P\left(\liminf _{t \rightarrow \infty}\left\|\gamma(t) Z_{t}-g\right\|=0\right)=1 .
$$

So, we proved that a.s. every point of $H^{1}$ is a limit point of $\left\{\gamma(t) Z_{t}: t>0\right\}$ which completes the proof of Theorem 1.2.

Remark 3.1. (a) Theorem 1.2 generalizes easily to processes $L=\{L(t): t \geqslant 0\}$ of the form (3), where $J \neq 0$ is an arbitrary skew-symmetric $m \times m$ matrix and $B$ an $m$-dimensional Brownian motion.

(b) The analogue of Theorem 1.1 when $\mathscr{C}_{0}^{1}$ is endowed with a Hölder norm has been proved by Baldi and Roynette (1992b). It might be interesting to investigate such a setting for the assertion of Theorem 1.2.

\section{Acknowledgements}

Research supported by the Natural Sciences and Engineering Research Council of Canada and by the Fonds FCAR of the Province of Quebec.

The authors also wish to thank the referees for their helpful comments on an earlier version of this paper.

\section{References}

Azencott, R. (1980). Grandes déviations et applications. In R. Azencott, Y. Guivarc'h and R.F. Gundy (eds), École d'Été de Probabilités de Saint-Flour VIII 1978, pp. 1-176. Lecture Notes in Math. 774, New York: Springer-Verlag.

Baldi, P. (1986) Large deviations and functional iterated logarithm law for diffusion processes. Probab. Theory Related Fields, 71, 435-453.

Baldi, P. and Roynette, B. (1992a) Entre la normalisation de Strassen et celle de Chung. C. R. Acad. Sci. Paris, Ser. I, 314, 935-940.

Baldi, P. and Roynette, B. (1992b) Some exact equivalents for the Brownian motion in Hölder norm. Probab. Theory Related Fields, 93, 457-484.

Berthuet, R. (1979) Loi du logarithme itéré pour certaines intégrales stochastiques. C. R. Acad. Sci. Paris, Ser. A, 289, 813-815.

Gaveau, B. (1977) Principe de moindre action, propagation de la chaleur et estimées sous-elliptiques sur certains groupes nilpotents. Acta Math., 139, 95-153.

Helmes, K. (1986) The "local" law of the iterated logarithm for processes related to Lévy's stochastic area process. Studia Math., 83, 229-237.

Helmes, K., Rémillard, B. and Theodorescu, R. (1986) The Functional Law of the Iterated Logarithm for Lévy's Area Process, pp. 338-345. Lecture Notes in Control Inf. Sci. 96. New York: SpringerVerlag.

Lévy, P. (1949) Le mouvement Brownien plan. Amer. J. Math., 62, 487-550.

Lévy, P. (1954). Théorie de l'Addition des Variables Aléatoires. Paris: Gauthier-Villars. 
Liptser, R.S. and Shiryaev, A.N. (1977) Statistics of Random Processes, Vol. 2. New York: SpringerVerlag.

Rémillard, B. (1994) On Chung's law of the iterated logarithm for some stochastic integrals. Ann. Probab., 22, 1794-1802.

Received October 1994 and revised December 1996 